\title{
Trans abdominal Ultrasonography in Acute Pancreatitis: a cross sectional study
}

\author{
Merina Gyawali', Ashish Shrestha ${ }^{2}$, Prakash Sharma ${ }^{3}$, Binod Bade Shrestha ${ }^{4}$, Subash Bhattarai ${ }^{5}$ \\ ${ }^{1}$ Assistant Professor, Department of Radio diagnosis and Imaging ${ }^{2}$ Resident, Department of Radio diagnosis and Imaging \\ ${ }^{3}$ Associate Professor, Department of Radio diagnosis and Imaging ${ }^{4}$ Associate Professor, Department of Surgery \\ ${ }^{5}$ Associate Professor, Unit of Gastroenterology, Department of Medicine \\ Manipal College of Medical Sciences, Pokhara, Nepal \\ ABSTRACT

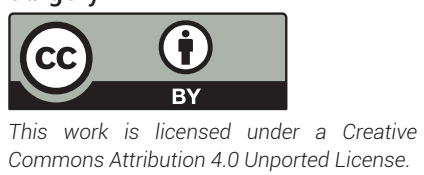

\section{BACKGROUND}

Acute pancreatitis (AP) is a common cause of acute pain abdomen. Contrast-enhanced Computed Tomography (CECT) of the abdomen is the imaging method of choice in acute pancreatitis. Ultrasonography can be used as the first, easily available imaging modality for the assessment of the pancreas. This study aims to study the transabdominal USG findings in patients with acute pancreatitis. It will also compare USG findings with CT findings in acute pancreatitis.

\section{METHODS}

A hospital-based cross-sectional, prospective study comprising of consecutive 55 patients with acute pancreatitis was conducted over a study period of 15 months. Trans abdominal USG findings and CECT abdominal findings in acute pancreatitis were studied and compared. Data analysis was done using SPSS version 20 and a $p$-value of $\leq 0.05$ was considered significant.

\section{RESULTS}

Pancreas was visualized by USG in only $69 \%$. Ultrasonography had some pancreatic and/or extrapancreatic findings in patients with acute pancreatitis in $84.2 \%$ of patients in whom the pancreas was visualized, whereas, it was $98.2 \%$ by CECT abdomen. USG was unable to demonstrate findings in $75 \%$ of patients with mild acute pancreatitis.

\section{CONCLUSION}

Transabdominal ultrasonography detection of pancreatitis was inferior to the CECT. It had a limited role in detecting mild acute pancreatic cases. Nonetheless, detection of etiological factor such as gallstones, and assessment of extra pancreatic fluid collection like ascites and pleural effusion were better visualised with ultrasound. USG is readily available, cheap, noninvasive, and can be utilized as an initial diagnostic tool for acute pancreatitis and ruling out other causes of acute abdomen.

\section{KEYWORDS}

Acute pancreatitis; contrast-enhanced computed tomography; ultrasonography

\section{INTRODUCTION}

Acute pancreatitis (AP) is an important cause of acute abdominal pain presenting to the emergency department. ${ }^{1,2}$ According to the American College of Gastroenterology and the revised Atlanta Classification, the presence of at least two of the following three features are required to diagnose AP. 1) characteristic abdominal pain, 2) elevation of pancreatic enzymes in the serum to at least three times the upper limit of normal, and 3) specific computed tomography (CT) findings (but may also include transabdominal ultrasonographic or magnetic resonance findings).3,4

Trans abdominal ultrasonography and CT abdomen are commonly used diagnostic imaging modalities for acute pain abdomen including acute pancreatitis. Ultrasonography provides the first inexpensive, non-invasive, easily available, and radiation-free imaging of the pancreas. ${ }^{5}$ However; it has limitations in obese patients and in those with large amounts of bowel gas. ${ }^{6}$

Contrast-enhanced CT (CECT) with the use of intravenous (IV) contrast agent is the imaging method of choice in acute pancreatitis which is highly accurate and sensitive than USG

\section{*Corresponding Author}

Dr Merina Gyawali, Department of Radio diagnosis and Imaging

Manipal College of Medical Sciences and Teaching Hospital, Pokhara, Nepal Email: drmerinag@gmail.com | Mobile No: +9779807265511 
in both diagnosing as well as demonstrating the extent. ${ }^{7}$ It is useful in the assessment of pancreatic necrosis, as well as inflammatory changes and local and/or extra pancreatic complications. ${ }^{8,9}$.

In primary health care centers, where CT is not available, transabdominal USG may be the only tool available for the evaluation of patients with acute pain abdomen suspected of acute pancreatitis. This study is undertaken to describe the trans abdominal USG findings in patients with acute pancreatitis and compare them with the findings of CECT abdomen.

\section{METHODS}

This prospective, cross-sectional, hospital-based study was carried out in the department of Radiodiagnosis and Imaging at Manipal College of Medical Sciences and Teaching Hospital, Nepal from June 2020 to August 2021 for duration of 15 months. Acute pancreatitis was diagnosed when two out of three features were present as per Atlanta classification.

Convenience sampling method was used. All patients aged $\geq 18$ years who presented with acute abdominal pain suggestive of acute pancreatitis were enrolled for the study. Patients with chronic pancreatitis, pancreatic malignancy, pregnant females, patients with renal insufficiency (creatinine $>1.5$ ), history of blunt abdominal trauma, those patients who didn't undergo CT abdomen, those with incomplete records, and those who failed to give consent were excluded from the study.

A detailed history was taken from the patient or patient relatives and physical examination was carried out. All relevant laboratory reports were collected. Ultrasound of the abdomen was done by second/third-year residents at emergency at the time of presentation and repeated after 72 hrs by a consultant Radiologist. Ultrasound examinations were performed WIPRO GE Logiq P3, Ultrasound, USA Inc. The normal pancreas appears homogeneous and isoechoic or hyperechoic to the normal liver on ultrasonography. Enlarged or bulky, hypo echoic, and heterogeneous pancreas on ultrasonography suggest pancreatic inflammation and was considered diagnostic of acute pancreatitis. The size of the normal pancreas in adults is up to $3 \mathrm{~cm}$ for the head, $2.5 \mathrm{~cm}$ for the neck and body, and $2 \mathrm{~cm}$ for the tail. ${ }^{6}$ The enlarged or bulky pancreas was quantified with exceeding diameters, with marked anterior bowing and surface irregularity. Contrast-Enhanced Computed Tomography (CECT) abdomen was usually done after 3-5 days of admission. The CT scan used was PHILIPS Ingenuity 128 CT scanners, NETHERLANDS Inc. Modified CT Severity Index (CTSI) was used as CT diagnostic and prognostic criteria for acute pancreatitis. Patients were classified into mild, moderately severe, and severe acute pancreatitis based on the revised Atlanta classification (RAC). ${ }^{3}$
Data were collected covering the relevant parameters for the study. All categorical data were expressed in percent and absolute numbers. All numerical continuous data were expressed in mean $\pm S D$. Chi-squared test or Fisher's exact test (wherever Chi-squared test was not feasible) were used to compare the significant differences of proportions for categorical data. All tests were analyzed with a 95\% confidence interval and they were considered statistically significant if the $p$-value was $\leq 0.05$. Data analysis was done using Statistical Packages for the Social Sciences (SPSS) 20. Informed consent was taken from the patient/patient's relative. Study approval and ethical clearance were obtained from the institutional review committee.

\section{RESULTS}

A total of 67 patients were diagnosed with acute pancreatitis during the study period. However, seven patients didn't undergo CT scan of abdomen and further five were excluded because of inadequate data. Finally, a total of 55 patients, comprising of 33 (60\%) males and 22 (40\%) females were taken up for the study ( $M: F=3: 2)$.

The mean age of subjects was $43 \pm 9.75$ years with a range of $24-67$ years of age. Patients were classified as per sex and age groups (Table 1$)$. Majority ( $n=35 ; 63.7 \%)$ of cases were aged between 41-50 years of age. There was no gender difference in the occurrence of acute pancreatitis among different age groups ( $p=0.89 ; p>0.05)$.

Table 1: Age/ sex distribution of study subjects $(n=55)$

\begin{tabular}{|l|c|c|c|}
\hline \multicolumn{1}{|c|}{ Sex } & Male & Female & Total \\
\hline$\leq 30$ years & 3 & 2 & 5 \\
\hline $31-50$ years & 23 & 15 & 38 \\
\hline $51-70$ years & 7 & 5 & 12 \\
\hline Total & 33 & 22 & 55 \\
\hline
\end{tabular}

The commonest etiology of pancreatitis was alcohol, seen in 32 (58.2\%) followed by biliary pancreatitis in 15 (27.2\%) cases. Pancreatitis due to hypertryglyceridemia was seen in $4(7.3 \%)$ and in rest $4(7.3 \%)$ cases, etiology was obscure.

Pancreas was visualized in only 69\% (38 out of 55 patients) by transabdominal USG. The USG findings in patients with acute pancreatitis were as in Table 2.

Table 2: Imaging findings in study subjects $(n=55)$

\begin{tabular}{|l|l|l|}
\hline \multicolumn{1}{|c|}{ Parameters } & USG & CT SCAN \\
\hline Visualization of pancreas & 38 & 55 \\
\hline Enlarged pancreas & 36 & 54 \\
\hline Heterogeneous appearance & 32 & 54 \\
\hline Gall stones & 15 & 11 \\
\hline
\end{tabular}




\begin{tabular}{|l|l|l|}
\hline CBD stone & 3 & 2 \\
\hline Acute fluid (peri pancreatic fluid) collection & 3 & 5 \\
\hline Pleural effusion & 12 & 10 \\
\hline Ascites & 7 & 7 \\
\hline
\end{tabular}

Among the 38 patients with visualized pancreas, 32 (84.2\%) were detected with bulky, hypo echoic and heterogeneous pancreas (Figures 1 and 2).

Pancreas was visualized in all the cases evaluated by CT abdomen. Fifty four (98.2\%) cases showed some form of pancreatic, peri pancreatic inflammation, peri pancreatic fluid collection, and/or hypo density in CT abdomen (Fig 3). Pancreatic necrosis was evident in 13(21\%) patients with contrast enhanced CT abdomen (Fig 4). The other CT findings were as in Table 2. Modified CTSI score was 0-2 (mild pancreatitis) in 24 (35.5\%) patients, 4-6 (moderate pancreatitis) in $21(45.1 \%)$ patients and 8-10 (severe pancreatitis) in 10 (19.4\%) patients.

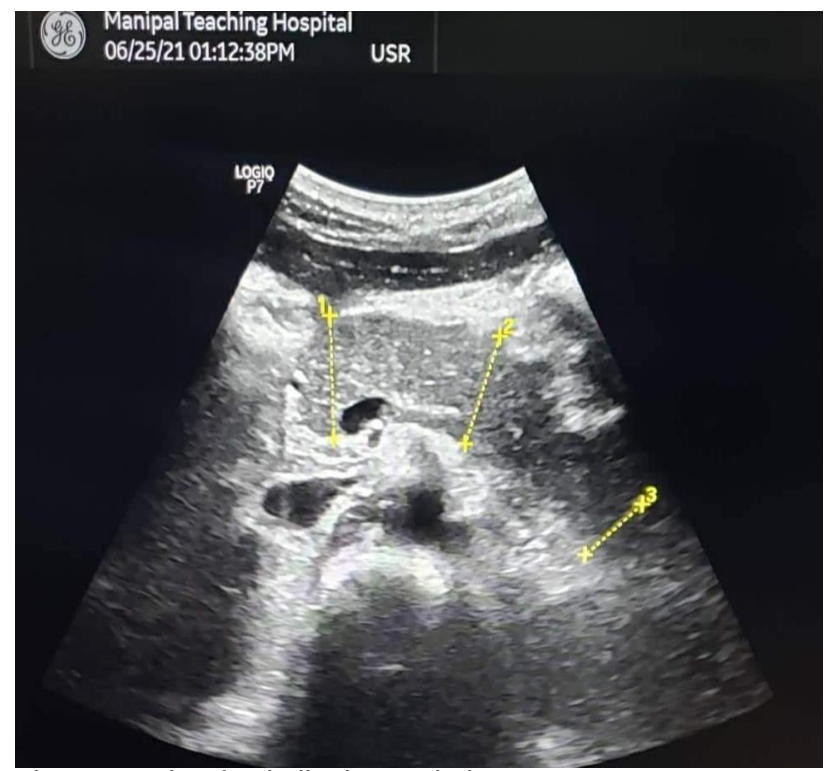

Fig 1: USG showing bulky, hypoechoic pancreas

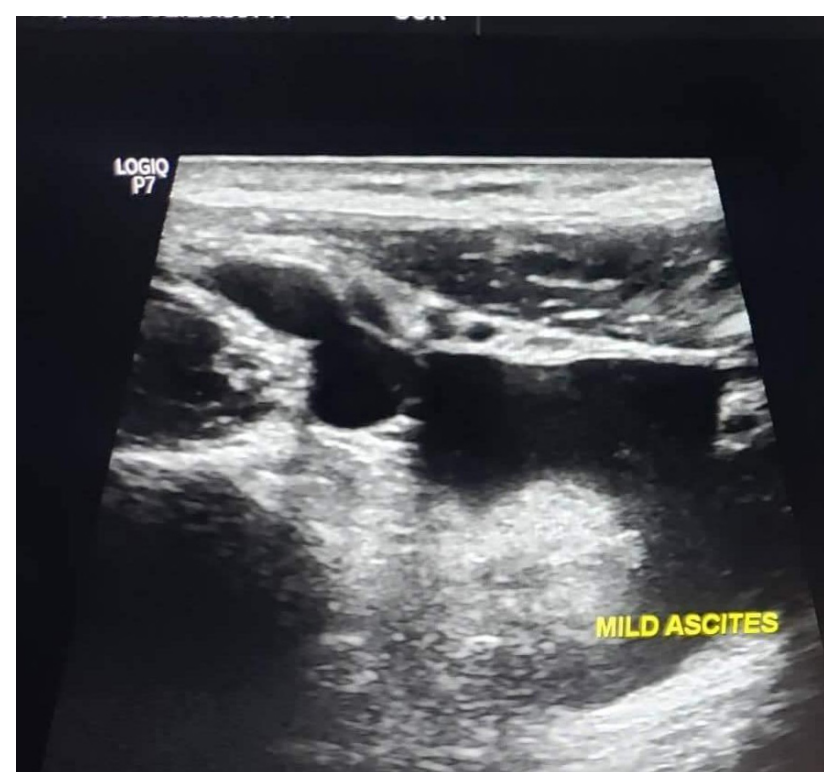

Fig 2: USG showing mild ascites

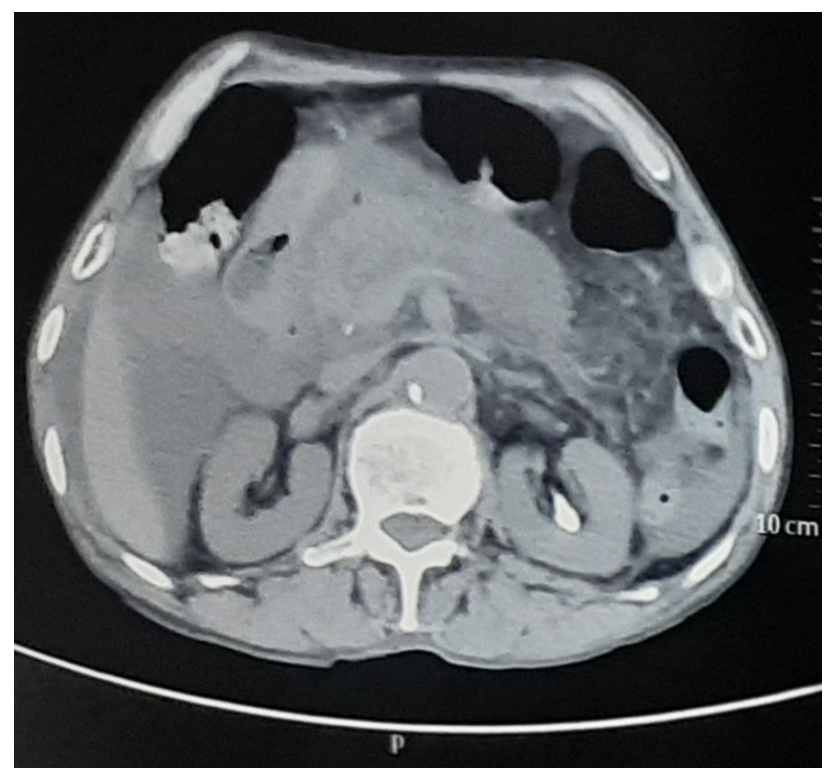

Fig 3: CT axial section showing bulky pancreas with peri pancreatic fluid and ascites

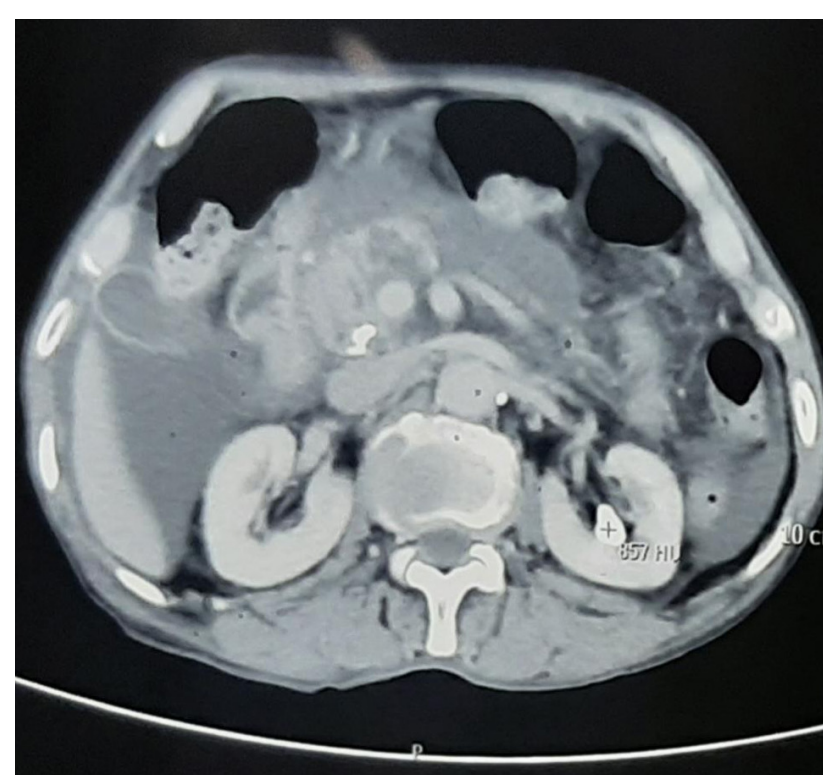

Fig 4: CECT axial section showing pancreas body and tail with $>30 \%$ necrosis 
Ultrasonography detected findings suggestive of acute pancreatitis in all patients with severe pancreatitis (modified CTSI score $\geq 6$ ). But USG was unable to demonstrate findings in 18 out of $24(75 \%)$ patients with mild acute pancreatitis (modified CTSI score $\leq 2$ ) and in 5 out of 21 (23.8\%) patients with moderately severe pancreatitis (CTSI score 4-6).

Ultrasonography detected acute pancreatitis in $84.2 \%$ of patients in whom the pancreas was visualized, whereas, it was $98.2 \%$ by CECT. This difference in detection of acute pancreatitis in USG and CECT was however not statistically significant $(p=0.58 ; p>0.05)$. Gall stones were better visualized by USG (15 vs. 12 by CT). Ultrasonography detection of biliary stone and pleural effusion was higher than those with CECT. Peripancreatic fluid collections were best studied by the CECT abdomen when compared to USG (Table 2).

\section{DISCUSSION}

The mean age of subjects was $43 \pm 9.75$ years with male predominance ( $M: F=3: 2)$ in the current study. Male predominance with the majority of patients in the age group of 41-50 years at presentation was consistent with recently published studies by Bhatt et al. ${ }^{10}$, Raghuwanshi. ${ }^{11}$ and Bhati et al. ${ }^{12}$

The pancreas was not visualized by USG in $31 \%$ in the current study. This was in consistent with the finding by Bhati et al. ${ }^{12}$, where, $37.21 \%$ of the pancreas was not visualized. Ultrasonographic evaluation gets hampered in $25 \%-30 \%$ of patients with acute pancreatitis due to over lapping gastrointestinal gas. ${ }^{13}$ Ultrasonography imaging fails to delineate pancreas during periods of paralytic ileus in acute pancreatitis. It lacks the detailed characterization of pancreatic parenchyma and is unable to delineate the extent of pancreatic necrosis. ${ }^{14}$ Pancreas may appear normal in USG in cases with mild acute pancreatitis. ${ }^{15,16}$ USG was unable to demonstrate pancreatic inflammation in $75 \%$ of patients with mild acute pancreatitis in the current study. Similar results were reported by Bhatt et al. ${ }^{10}$ and Karwa et al. ${ }^{17}$

USG is the first line of modality for the confirmation of acute pancreatitis and excluding other causes of acute abdomen. It can detect gallstones which is a common cause of acute pancreatitis. ${ }^{16}$ USG biliary stone detection was superior to CT abdomen in the current study. These findings were also supported by previously published studies. ${ }^{10,12,13,15}$

Visualization of the pancreas is not interfered by the overlying bowel gas in CT abdomen. It allows for precise information about the size, parenchyma appearance of the pancreas, peripancreatic region, presence of pseudo cyst, and even pancreatic necrosis. ${ }^{15}$ Computed tomography of abdomen, not only establishes the diagnosis of acute pancreatitis but allows staging the severity of the disease and its prognosis. 18 Modified CTSI score revealed mild pancreatitis in $35.5 \%$, moderate pancreatitis in $45.1 \%$, and severe pancreatitis in $19.4 \%$ in the current study. These findings were in consistent to CECT findings by Bhati et al. 12

Trans abdominal USG detected acute pancreatitis in $84.2 \%$ of patients in whom the pancreas was visualized, whereas, it was $98.2 \%$ by CECT in the current study. USG and CT detection of pancreatic inflammation was $84 \%$ and $100 \%$ by Lalith et al. ${ }^{19}$, finding almost similar to our study. CT scan was far better and it efficiently detected pancreatic pathology and complications in the studies by Tomislav et al. ${ }^{15}$, Karwa et al ${ }^{17}$ and Gupta et al. ${ }^{20}$

\section{CONCLUSIONS}

Ultrasound of the abdomen can detect and characterize the size and echotexture of the pancreas. USG detected the etiological factor like gall stones, and extra pancreatic complications like ascites and pleural effusion even better than CT. Ultrasonography detection of pancreatitis was, however, inferior to the CECT. It had a limited role in detecting mild acute pancreatic cases. However, USG being readily available, cheap, and non-invasive, is a very helpful initial tool for diagnosis of acute pancreatitis, evaluating the gallbladder and biliary tract, detecting fluid collections, and ruling out other causes of acute abdomen.

\section{LIMITATIONS OF THE STUDY}

This was a single-center study and the sample size was small. Ultrasonography findings are user-dependent. Excessive gas during acute pancreatitis always provides a poor window for USG evaluation. The pancreas was not visualized by ultrasonography in all subjects under study.

\section{CONFLICT OF INTEREST: NONE}

\section{SOURCE OF FUNDING: NONE}

\section{REFERENCES}

1. Ragsdale L, Southerland L. Acute abdominal pain in the older adult. Emerg Med Clin North Am. 201 1; 29(2): 429-48

2. Calleja GA, Barkin JS. Acute pancreatitis. Med Clin North Am. 1993; 77(5): 1037-56.

3. Thoeni RF. The revised Atlanta classification of acute pancreatitis: its importance for the radiologist and its effect on treatment. Radiology. 2012; 262(3): 751-64.

4. Banks PA, Bollen TL, Dervenis C, Gooszen HG, Johnson CD, Sarr MG,et al. Acute Pancreatitis Classification Working Group. Classification of acute pancreatitis--2012: revision of the Atlanta classification and definitions by international consensus. Gut. 2013; 62(1): 102-11.

5. Bennett GL, Hann LE. Pancreatic ultrasonography. Surg Clin North Am. 2001; 81(2): 259-81 


\section{Nepal Mediciti Medical Journal}

6. Johnson CD, Besselink MG, Carter R. Acute pancreatitis. BMJ 2014 (Clinical research ed.). 349: g4859.

7. Türkvatan A, Erden A, Türkoğlu M A, Seçil M, Yener Ö. Imaging of acute pancreatitis and its complications. Part 1: Acute pancreatitis. Diagn Interv Imaging. 2015; 96(2): 151-60

8. Mortele KJ, Wiesner W, Intriere L, Shankar S, Zou KH, Kalantari BN,et al. A modified CT severity index for evaluating acute pancreatitis: improved correlation with patient outcome. AJR Am J Roentgenol. 2004; 183(5): 1261-5.

9. Shyu JY, Sainani NI, Sahni VA, Chick JF, Chauhan NR, Conwell $D L$, et al. Necrotizing pancreatitis: diagnosis, imaging, and intervention. Radiographics. 2014; 34(5):1218-39.

10. Bhatt A, Tiparse A, Patel A, Gandhi B. USG and CT scan evaluation of patients of acute and chronic pancreatitis- a cross-sectional, comparative study. Int J Res Med Sci. 2017; 5(8): 3713-6

11. Raghuwanshi S, Gupta R, Vyas MM, Sharma R. CT Evaluation of Acute Pancreatitis and its Prognostic Correlation with CT Severity Index. Journal of Clinical and Diagnostic Research, 2016; 10(6): TC06-TC11.

12. Bhati $V$, Mital $M$, Verma $S R$, Sagar $S$, SharmaY, Singh AP. Evaluation of acute pancreatitis by USG and CT. EJPMR. 2019; 6(4): 496-503.

13. Minniti S, Bruno C, Biasiutti C, Tonel D, Falzone A, Falconi M, et al. Sonography versus helical CT in identification and staging of pancreatic ductal adenocarcinoma. J Clin Ultrasound. 2003; 31(4):175-82

14. Bertolotto M, D'Onofrio M, Martone E, Malagò R, Pozzi Mucelli R. Ultrasonography of the pancreas. Doppler imaging. Abdom Imaging. 2007; 32(2): 161-70.

15. Tomislav T, Saša G, Aleksandar N. Correlation of Clinical, Ultrasound and CT Findings in Patients with Acute Pancreatitis. Austin J Gastroenterol. 2014; 1(2): 1007.

16. Burrowes DP, Choi HH, Rodgers SK, Fetzer DT, Kamaya A. Utility of ultrasound in acute pancreatitis. Abdom Radiol (NY). 2020; 45(5):1253-64

17. Karwa MS, Lakhkar LD, Kachewar GS, Jethlia K. Role of Ultrasound and Computed Tomography Scan in Diagnosis of Acute Pancreatitis and Its Complications. Sch J App Med Sci. 2018; 6(7): 2840-48.

18. Balthazar EJ, Robinson DL, Megibow AJ, Ranson JH. Acute pancreatitis: value of CT in establishing prognosis. Radiology. 1990; 174(2): 331-6.

19. Lalith S, Ilangovan G. Comparative study of ultrasonography and computed tomography in diagnosis of acute pancreatitis. International Journal of Contemporary Medicine Surgery and Radiology. 2019; 4(3): 28-33.

20. Gupta S, Mittal A, Arion R K, Singal R. Comparative evaluation of Ultrasonography and Computed Tomography in pancreatic Lesions. J Medicine 2016; 17(2): 66-78 